Review Article

\title{
OVERVIEW OF FORMULATION, MANUFACTURE AND APPLICATIONS OF MINI TABLETS
}

\author{
PRATIKSHA PATIL ${ }^{*}$, RASHMI P. ${ }^{2}$
}

1,2Department of Quality Assurance, Acharya and B M Reddy College of Pharmacy, Soldevanahalli, Bangalore 560107 Karnataka, India Email: pratikshap194@gmail.com

Received: 13 Jun 2020, Revised and Accepted: 16 Aug 2020

\section{ABSTRACT}

Mini tablets, recent trend of solid dosage form, made remarkable contribution to avoid certain obstacles in people's mind and offered best therapeutic benefits, flexible dose and combined released pattern. Mini tablets were developed and reported as patient friendly with increased patient acceptance. Mini tablets were made into modified release system for better dose and prolong drug release. They offer the advantage of reduced dose dumping and increased effect of drug by localization. Manufacture of mini tablets are similar to conventional tablets but need a change in tooling, equipment and specifications. Evaluation parameters found to be similar with conventional tablets but doses are variable. Mini tablets can also be encapsulated and hence different drug combinations are tried and found useful.

Keywords: Mini tablets, Oral dosage forms, Manufacturing, Dose, Multi tooling punches, Capsule

(C) 2020 The Authors. Published by Innovare Academic Sciences Pvt Ltd. This is an open access article under the CC BY license (http://creativecommons.org/licenses/by/4.0/) DOI: http://dx.doi.org/10.22159/ijcpr.2020v12i5.39774. Journal homepage: https://innovareacademics.in/journals/index.php/ijcpr

\section{INTRODUCTION}

Conventional solid oral dosage forms like tablets, capsules, powder, lozenges are generally administered to the patients through oral route andare expected to deliver the drug at the target site in a shortest possible time. The onset and duration of any drugs administered depends on its route of administration, site of action therapeutic index and half-life. Among several problems involved with the formulation and use of conventional solid dosage forms, interval for dosing was found far shorter than the drugs half-life, resulting in numeral limitations. Some of other issues related to a conventional dosage form are like this kind of dosage form leads to poor medical adherence of patient which leads to increase the risk of losing a dosage of short-lived medication that requires frequent administration. Such dosage forms were found tough to achieve a stable state from the previously obtained plasma concentration time profile. Sometime fluctuation in the drug level will result improper medication which may leads to adverse effects [1].

Though conventional unit dosage forms were being used effectively since decades, its wide range of variations in blood stream concentration and its consequences signposted the need for improvement and innovation in solid unit dosage form [2].

Solid dosage forms used for controlled release can be considered as, Forms of single unit dosages (SUDFs) like capsules, coated or uncoated tablets and other forms are Mini tablets, pellets, granules are the (MUDFs) multiple forms of unit dosage $[3,4]$.

Since last decade, mini tablets which are one of multiple unit dosage forms considered as more convenient because they aided the ease of administration. Mini tablets found commonly encapsulated in capsules, sometimes in sachets. They can also be compressed into bigger tablets. These tablets were found ranging from 1.0 to $3.0 \mathrm{~mm}$ in diameter. They are intended to deliver and maintain desired concentration of medicament at particular site [4].

Mini tablets have various applications based on target sites and patient requirements [5]. They are used for/as pediatrics, gastro retentive, bio adhesive, $\mathrm{pH}$ responsive, orally disintegrating and biphasic mini tablets.

\section{Pediatric mini tablets}

Since syrups and tablets can be easily administered, they are the widely used for children. These kind of dosage forms exhibit various issues related to organoleptic characters, physicochemical properties, drug release pattern during formulation. Also it was found that it is difficult to adjust the dose since many parents are practiced to break tablet half and use for their children. This practice sometimes may lead to improper dosage and function of respective drug. Patient compliance is also one of the issues with conventional dosage form [6]. Viviane klinmann along with coworkers conducted clinical trials on newborns and preschoolers to assess mini tablets' acceptance in comparison with syrup. Results indicated the superior acceptance of uncoated mini tablets in comparison to syrup in total patient population of 306 children (with different proportions $14.8 \%$, 95\%, CI 10.2-19.4; $\mathrm{P}<.0001$ ). The level of swallowing capability for uncoated mini tablets was also found higher among children of 6 mo to $6 \mathrm{y}$ of age and found more suitable than liquid formulations [7]. Natalie spomer and team conducted clinical trials in young children to check that uncoated mini tablets are accepted in very young age group. The finding of a prospective cross-over study concluded that uncoated mini tablets are very promising substitute to fluid formulation and can be used in pediatrics drug therapy. Mini tablets were absolutely swallowed by specimens (6-12 mo of age) and they were able to handle them much better than the fluid formulation. Tablets were chewed by children between 2 and 4 y of age, but still handled quite well. Overall, acceptance rate of min tablets was found superior than expected [8]. Therefore usage of mini tablets for pediatrics was found more useful.

\section{Gastro retentive/floating mini tablets}

These kind of dosage forms are designed for extended release of the medication in the stomach and formulated by using agents which produce gas in the tablets so that they float on gastro intestine fluid. Sodium bicarbonate $\left(\mathrm{NaHCO}_{3}\right)$ or calcium carbonate $\left(\mathrm{CaCO}_{3}\right)$ (gas generating agents) coating, eudragits coating instead of swelling polymers were used in mini tablets to improve drug loading in the formulation. Mini tablets coating was done by using Fluid bed processor [9]. Sally A. El-Zahaand others designed gastro retentive levofloxacin as tablets-in-capsule in floating form and evaluated conducted method for the development and evaluation of the mini tablets in capsule floating and evaluated antibacterial action against Helicobacter pylori. Study results have shown better physicochemical properties and floating parameters. Optimized formulation exhibited floating time lag less than $1 \mathrm{~s}$ and maximum floating time was more than $24 \mathrm{~h}$. Volunteers held floating mini tablets of levofloxacin having polymer HPMC K100 M in the stomach for over $4 \mathrm{~h}$ was proved as better alternate for the eradication of $\mathrm{H}$ pylori [10].

\section{Bio adhesive mini tablets}

These mini tablets are used to administer medication through vaginal route reliably for longer duration. Division of mini tablets 
were done into several units so that they get spread uniformly in vaginal cavity covering maximum vaginal epithelium. These tablets released the drug by swelling and forming micro-gels in a steady manner with optimal bioavailability. This type of mini tablets have been developed by Hiorth $\mathrm{M}$ and colleagues and found that mini tablets with either HPMC or HPC matrix possessed adequate mechanical strength, greater bio adhesive actions along with $\mathrm{pH}$ independent release of prototype drug. Results indicated that both formulations can be considered for women's treatment. Therefore these mini tablets can be used to have better interaction with mucosal tissue and sustained drug release [11].

\section{pH responsive mini tablets}

Human GIT pH differs considerably. If drug absorption is more at any one site, selected drug dissolution can be easily accomplished by coating with polymer like Eudragits which is a form of $\mathrm{pH}$ responsive release [12]. H. N. Shivakumar and coworkers designed and developed a pH sensitive mini tablet formulation and carried out estimation of chronotherapeutic theophylline delivery and concluded that the $10 \%$ of coating weight gain was found enough to convey gastro-resistant property to release drug at higher $\mathrm{pH}$ value. $\mathrm{pH}$ sensitive polymer can be used to establish a colon-specific drug delivery system successfully. Pulsed release observation in most of the cases was found highly desirable for targeting a multi-unit systems nocturnal peak symptoms of asthma at night [13].

\section{Biphasic mini tablets}

These kind of tablets includes a quick release component and a slow release portion for two parts. First part of the drug releases immediately with the administration of the drug and other part of the drug gets released slowly in a controlled mode. This form was found useful inanti-hypertensive drugs where repeated dosing could be avoided. Specific medications can be packed into mini tablets to treat various diseases in the same capsules. Carla M. Lopes et al., conducted an experiment where mini tablets were compressed into biphasic drug delivery system using different composition of (HPMC or EC) at in order to achieve different drug release. 10 or $21 \mathrm{mini}$ tablets were used to study release profile of drug and to analyze whether it depends on the number and composition of the subunits. Hydroxypropyl methyl cellulose mini-tablets disintegrated at a constant rate within a fraction of a second showing dissolution profile for a continuous time of eight hours. Mini-tablets powder release biphasic formulation causes some improvments in the process and duration of release of medication, which was found to be longer retaining in comparison to non-compressed mini-tablets [14].

\section{Oral disintegrating mini tablets}

The latest type of dosage that quickly disintegrates into the mouth without chewing upon oral administration within 1-3 min, unlike any other form of solid oral dosage. These tablets shown various characters without additional water as they disintegrated in mouth. After disintegration tablet had become soft paste or liquid suspension and gave better acceptance of patient. M. El-Say with others developed orally disintegrating Risperidone mini tablets a robust pediatrics drug product and concluded that the optimized oral disintegrating mini tablets of $2 \mathrm{~mm}$ diameter, suitable mechanical strength, minimal friability, acceptable weight, drug uniformity, and short disintegration time were prepared and reported [15].

\section{Following are the other advantages of mini tablets}

They had shown low inter and intra-subject variability and less dose dumping risk. Mini tablets can be easily produced in the same size, weight, better uniformity, systematic shape and smooth surface. Such tablets provide the gastrointestinal tract with a high degree of dispersion and reduce the risk of high concentration of local medicines. Compared to pallets, such tablets do not need solvents for their processing, which can prevent stability issues [16]
Processing of pellets involves technically challenging process such as fluid bed granulation, extrusion or spheronization, on the other hand simple tableting procedure can be employed for the development of mini tablets [14]. With low porosity and high strength these tablets were found to retain more reproducible structure and shape than other multi particulate dosage forms [17]. Thus multi particulates, mini tablets showing potential applications as a flexible drug delivery system for various particulates.

\section{Pre formulation studies for mini tablets}

These studies deals with physicochemical and various pharmaceutical properties related to drugs and excipients and will provide an idea of any modification in the properties affecting the nature of drug product to get better results.

\section{Drug polymers compatibility studies [3]}

Studies of compatibility with drug polymers were conducted using FTIR. Drug compatibility with the excipients can be tested by using spectral analysis. Also this study help to carry out drug polymers study on individual pure drugs and physical mixture of the drug and additive.

Precompressional parameters like, Angle of repose, Bulk density and tapped density, Compressibility index and Hausner's ratio were performed for the powder blend to ensure the best powder flow property and achieve good end product.

\section{Tooling used in compression of mini tablets}

Normal tablet compression is performed using single tip tools that can be interchanged as needed. Mini tablets can be generally manufactured by compression using a rotary tablet press [18]. A special tools can be used for the mini tablets called multi-tip tooling. This can be mostly used for two reasons. First, this increases the output efficiency and reduces the powder dwelling time in the feed frame, which contributes to over-lubrication or separation of the powder. Secondly, by increasing the number of tips, the force applied to a pair of opposing punch tips is multiplied by the number of tips, resulting in a greater overall compression force that can be controlled more accurately. Multiple-tip punches tools offers multi-part assemblies and monoblock from tablet devices sellers. Monoblock punches provide quicker tool installation and easier cleaning, can be made with tighter tolerances and are more resistant to tip breakage than multi part tooling installed. Multi-tip tool must meet more stringent requirements for machining and mechanical stability compared to larger tablet tools and are more labor-intensive to manufacture, resulting in higher production costs [19]. Showing various multi tip punches used for compression in fig. 1, 2, 3 and mini tablets in fig. 4 below:

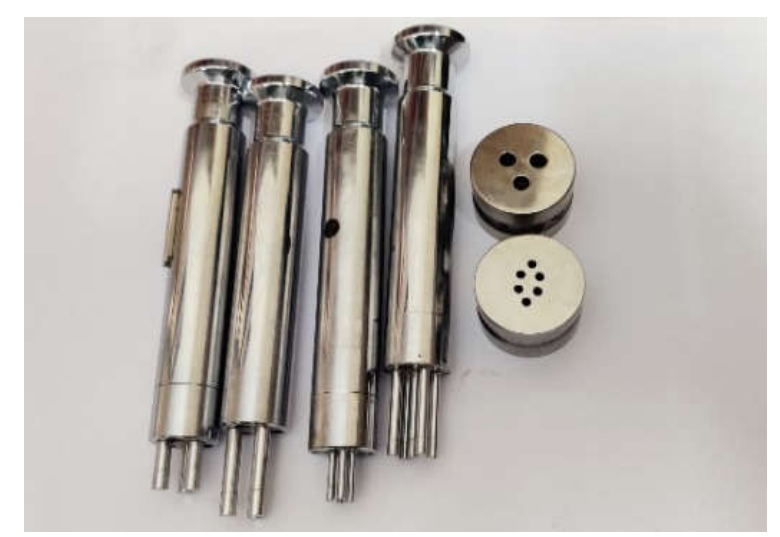

Fig. 1: It shows upper and lower multi-tip punches and die (with 3 and 5) tooling 


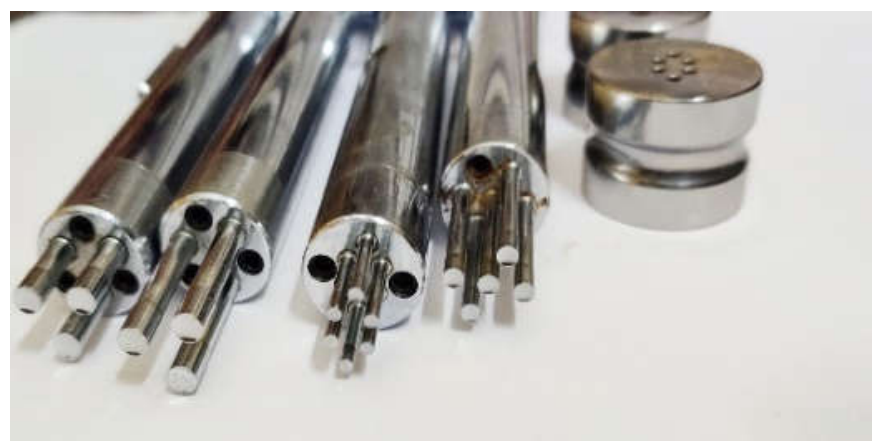

Fig. 2: It shows tip sizes of the multi tip punches/compression

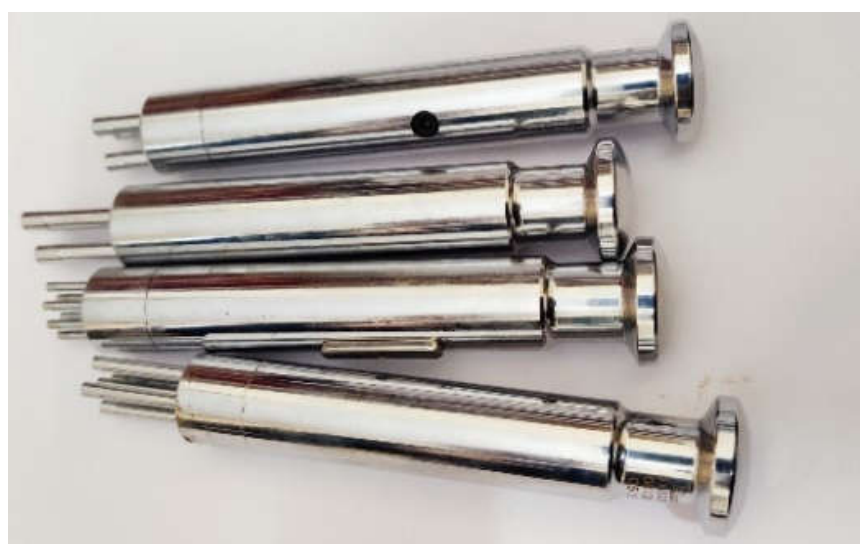

Fig. 3: It shows multi tip tablet punches

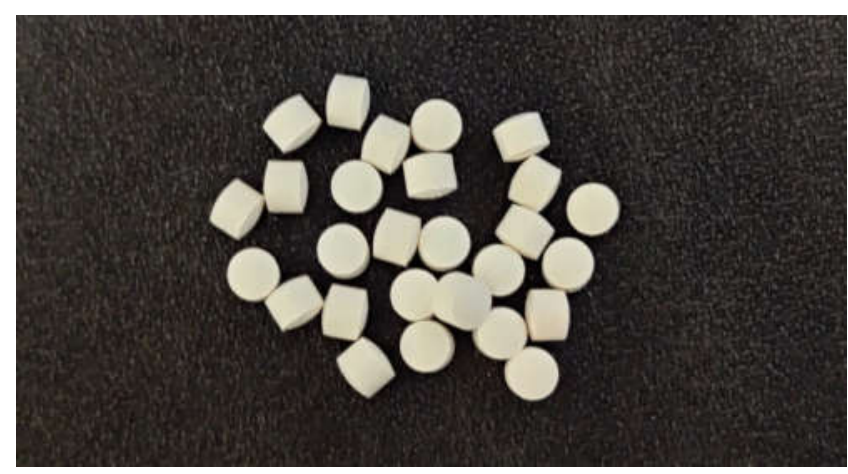

Fig. 4: It shows mini tablets

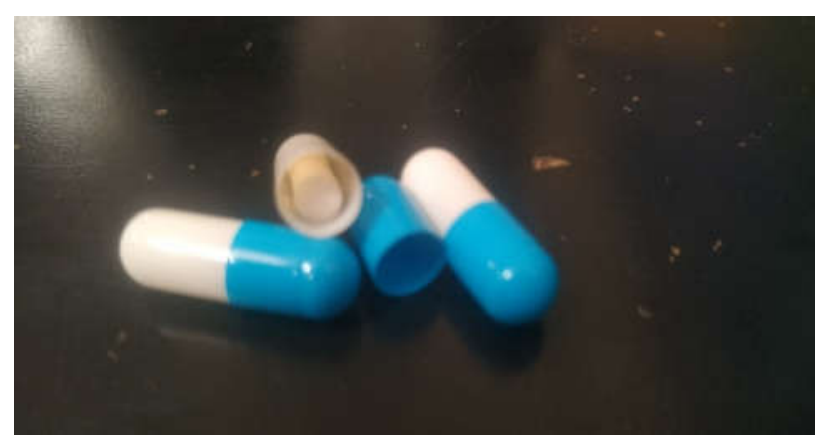

Fig. 5: Its shows mini tablets in capsule 


\section{Methods of manufacturing mini tablets [20]}

Different methods will be employed for the manufacturing of mini tables given below:

\section{Direct compression technique}

This is a process where the powder mixture holding API and excipients which can be compressed directly into biconvex mini tablets. Direct compression grades have been used to achieve the required hardness. Problems of stability were found lower than those of tablets with wet granulation.

\section{Dry granulation technique}

This technique is used to develop thermo-labile, moisture-sensitive tablets. A roller compactor or chilsonator processing equipment can be used for this techniques. This machine compresses under extreme pressure as premixed powders have been found in the shape of a brittle ribbon, board, or fragment between two counter spinning rollers. By employing 'slugging' techniques granules can be produced, where slug are screened or milled and granules are mixed and finally compressed with other excipients.

\section{Wet granulation}

This technique involves using binder solution to form granules which are further compressed into mini tablets.

\section{Melt-extrusion technique}

The premixed powder API and excipients is allowed to move towards melt-extruder. In the melt extruder material range, a speed of the screw, feed rate and temperature parameters can be set. Then the extrudates are milled and sieved. Using a compression tool, the granules collected are then compressed into mini tablets.

\section{Coating of compressed mini tablets}

Principles involved in coating for mini tablets are based on some objectives like: such tablets help to mask the drugs taste and odor also monitor the release of the drug form the tablet. To protect the drug product with an acid resistant enteric coating from the stomach environment. Providing concurrent release of medication. Use different color and contrasting printing to enhance medicinal appearance of the drug.

\section{Coating procedures for mini tablets}

Mini tablets are coated by using different techniques as sugar, film compression and enteric coating process.

Several polymers will be used for enteric coating of mini tablets such as Methacrylic acid or ethyl acrylate, Sodium alginate and stearic acid, Cellulose acetate succinate, Cellulose acetate trimellitate, Cellulose acetate phthalate, Hydroxy propyl methyl cellulose phthalate, Hydroxy propyl methyl cellulose acetate succinate [21].

Polymers used for the film coating of mini tablets like Polyox WSR 1105, microcrystalline cellulose 200, Colloidal silicon dioxide, Magnesium stearate [22]

Polymers used for the compression coating mini tablets such as Eudragits RS/RL 30D, Trietilcitrate, Talc,Titanium dioxide, Yellow quinolone, Pigment dispersion WAS, Water [23]

With different coating procedures suitable polymers can be used for the mini tablets coating.

Mini tablets are usually coated by using fluidized bed coaters or using modified coating pans with enteric coating polymers. Enteric coating is a polymer membrane that protect it against the acidic $\mathrm{pH}$ of the stomach when added to a drug and releases the medication in the small intestines alkaline environment. They cannot dissolved in the stomach acidic juices, but they break down in the small intestines alkaline setting. Drug which leads to mucosa inflammation or are inactivated in stomach can be covered with material that dissolves in the small intestine alone [24, 25]. Film coating is considered to produce an elegant product appearance. High stability against heat, light, moisture, air. High compatibility with other coating solution additives. Resistance to nontoxic pharmacological activity. Compatible to printing procedure [26, 27]

\section{Different formulations of mini tablets}

The three steps in the formulation process (mini-tablet-in-capsule systems) where first step is the Mini tablet formulation/manufacture, second step is coating of mini-tablets by using sufficient polymer coating, encapsulation of mini tablets were filled with hard gelatin or Hydroxypropylmetyl cellulose capsules of coated mini tablets. Third step is the preparation of mini tablets or granules into capsules systems; by using formula to calculate the immediate-release dose, $\mathrm{DL}=\mathrm{CmaxVd}$

Cmax = the maximum plasma concentration, and $\mathrm{Vd}$ is the distribution volume

Preparation of immediate release component (Granules) or immediate release coated mini tablets IRCMT

Due to its strong compaction and disintegration properties, the measured amount of medication and other appropriate excipients released immediately like (Microcrystalline cellulose [avicel PH 102]). Appropriate super disintegrants were used to get the medication release immediately. Wet granulation method will be used to prepare the granules. Also wet granulation techniques will be employed for the preparation of IRCMT

\section{Preparation of sustained-release coated mini-tablet (SRCMT)}

These tablets were developed by using same process which was employed for the immediate release coated mini tablets Hydroxypropyl methyl cellulose $5 \mathrm{cps} / 15 \mathrm{cps}$, ethyl cellulose, magnesium stearate, ethyl alcohol, and water have been used to prepare coating suspension. In the coating preparation, magnesium striate were used to reduce the friction between the mini tablet surfaces, the filling device and the capsules of HPMC

\section{Preparation of coated mini-tablet-in-capsule system}

Two IRCMT and three Sustained release mini tablets were placed in each HPMC capsule of size 1 in order to prepare the CMTICS. A similar or different SRCMT ratio is put in each HPMC capsule in order to obtain different sustained release profile of the CMTICS $[28,29]$.

\section{Evaluation of mini tablets $[30,31]$}

Mini tablet evaluation is like regular tablet evaluation, general tests are evaluated given below:

\section{Weight variation test}

Randomly 20 tablets were selected and weight of the individual tablets is noted. It calculates the average weight. Noted individual tablet weight should not be less than $90 \%$ and more than 110 percent of the average weight, as per USP.

\section{Hardness}

It is calculated and expressed in $\mathrm{kg} / \mathrm{cm}^{2}$ using Pfizer hardness tester Six tablets were picked randomly for hardness testing. From the each formulation mean and the standard deviation values will be determined.

\section{Thickness}

A digital caliper and screw gauge (Mitutoyodigimatic caliper) can be used for measuring mini tablets. This is expressed in the terms of $\mathrm{mm}$.

\section{Percentage friability}

Mini tablets are performed with the aid of Roche friabilator or veego friabilator. Normally twenty mini tablets of each lot are selected a random and the initial weight $\left(\mathrm{W}_{0}\right)$ is noted and transferred to friabilator. Allow the drum to rotate for $4 \mathrm{~min}$ at $25 \mathrm{rpm}$. With the aid of a soft brush, any loose dust was collected and mini tablets were weighed again $\left(\mathrm{W}_{1}\right)$.

\section{Drug content uniformity}

Using UV visible spectrophotometer, 5 mini tablets were weighed and they are grounded into a mortar and then accurately weighed 
powder containing $10 \mathrm{mg}$ of medicine transferred in $100 \mathrm{ml}$ volumetric flask. Find out the content uniformity of the drug by using suitable wavelength.

\section{In vitro disintegration}

Mini tablets with disintegration test apparatus will be assessed accordingly by using I. P conditions.

\section{In vitro dissolution studies}

These studies are performed using suitable buffer solution at specific rpm and temperature in USP type II dissolution testing apparatus for a definite period of time. All of these variables depend on the formulation. At the correct wavelength, $10 \mathrm{ml}$ of the test sample will be collected and studied by using Ultraviolet spectrophotometer. The drug release will be checked at the same temperature and rotational speed for a definite period of time. $10 \mathrm{ml}$ of the sample will be removed at all times $(15,30,60,90,120,20$ and $360 \mathrm{~min}$ ) and evaluated using a UV spectrophotometer.

\section{Stability studies [32]}

It is an essential part of the process of drug development and plays vital role in pharmaceutical product registering. This study were conducted in compliance with ICH guidelines and also it will help to track changes in product material consistency over time by taking into account temperature, humidity and light effects. Implementing storage conditions: $40{ }^{\circ} \mathrm{C} \pm 2{ }^{\circ} \mathrm{C} / 75 \% \mathrm{RH} \pm 5 \% \mathrm{RH}, 25{ }^{\circ} \mathrm{C} \pm 2{ }^{\circ} \mathrm{C} / 60 \%$ $\mathrm{RH} \pm 5 \% \mathrm{RH}$ for the period: $1,2,3$ mo.

\section{CONCLUSION}

The review focused on few applications of mini tablets along with various aspects of mini tablets manufacturing. Production of these tablets was found to be identical to standard normal tablets, but due to small dies it requires good powder flow property and precise control of process parameters and special care. Mini tablets were found to be more advantageous over single unit dosage forms and attuned alternative when compared to granules and pellets. They are patient friendly drug delivery system and hence evolved into a transformed release system (extended release, delayed release of colon, pulsatile and bi-modal release and gastro-retaining systems) which shows better bioavailability compared to unit single dosage form.

\section{ACKNOWLEDGMENT}

The author would like to thank the coauthor and Acharya and BM Reddy College of pharmacy Bangalore, Karnataka, India for supporting us in the literature review and drafting of manuscripts.

\section{FUNDING}

$\mathrm{Nil}$

\section{AUTHORS CONTRIBUTIONS}

All authors have contributed equally.

\section{CONFLICT OF INTERESTS}

Declare none

\section{REFERENCES}

1. https://www.researchgate.net/publication/299812709. [Last accessed on 09 Dec 2019].

2. Rao KV, Venkatchalam VV. Dissolution kinetics of immediate release mini tablets of cefuroxime axetil. W J Pharm Res 2018;7:923-46.

3. Patel H, Shaikh SN. Formulation development optimization and evaluation of telmisartan mini tablets. Int J Pharm Bio Sci 2018;8:545-54.

4. Chithaluru K, Tadikonda R, Gollapudi R, Kandula KKK Formulation and in vitro evaluation of sustained release matrix tablets of losartan potassium. Asian J Pharm Clin Res 2011;8:403-10.

5. Priyanka, Kumar K, Teotia D. A comprehensive review on pharmaceutical mini tablets. J D Delivery Ther 2018;8:382-90.
6. Singh S, Virmani T, Virmani R, Kumar P, Mahlawat G. Fast dissolving drug delivery systems: formulation, preparation techniques and evaluation. Universal J Pharm Res 2018;3:60-9.

7. Klinmann V, Spomer N, Lerch C, Stoltenberg I. Favorable acceptance of mini-tablets compared with syrup: a randomized controlled trial in infants and preschool children. J Pediatrics 2013;163:1728-32.

8. http://group.bmj.com/group/rights-licensing/permissions. [Last accessed on 09 Jan 2020]

9. Lingam M, Ashok T, Vekateswarlu V, Rao YM. Design and evaluation of a novel matrix type multiple units as biphasic gastroretentive drug delivery systems. AAPS PharmSciTech 2008;9:1253-61.

10. El-Zahaby SA, Kassem AA, El-Kamel AH. Design and evaluation of gastroretentive levofloxacin floating mini-tablets-in-capsule system for eradication of helicobacter pylori. Saudi Pharm J 2014;22:570-9.

11. Hiorth M, Nilsen S, Thol. Bioadhesive mini-tablets for vaginal drug delivery. Pharmaceutics 2014;6:494-511.

12. Ranjith $\mathrm{K}$, Mahalakshmi R. Pharmaceutical mini tablets. Int J Pharm Technol Res 2015;7:507-15.

13. Shivakumar HN, Suresh S, Desai BG. Design and evaluation of $\mathrm{pH}$ sensitive minitablets for chronotherapeutic delivery of theophylline. Indian J Pharm Sci 2007;69:73-9.

14. Lopes CM, Lobo JMS, Pinto JF, Costa P. Compressed mini-tablets as a biphasic delivery system. Int J Pharm 2006;323:93-100.

15. El-Say KM, Ahmed TA, Abdelbary MF, Ali BE, Aljaeid BM, Zidan AS. Risperidone oral disintegrating mini-tablets: a robustproduct for pediatrics. Acta Pharm 2015;65:365-82

16. Mahajan KV, Akarte AM, Sapate MK, Baviskar DT, Jain DK. Designing and evaluation of compressed mini-tablets of ramipril as a biphasic delivery system. Indo Am J Pharm Res 2013;3:7277-87

17. Thomson SA, Tuleu C, Wong IC, Keady S, Pitt KG, Sutcliffe AG. Min tablets: new modality to deliver medicines to preschool-aged children, Official. J Am Academy Pediatrics 2009;123:235-8.

18. https://epdf.pub/developing-solid-oral-dosage-formspharmaceutical-theory-and-practice.html. [Last accessed on 09 Jan 2020]

19. https://www.americanpharmaceuticalreview.com/FeaturedArticles/190921-Minitablets-Manufacturing-CharacterizationMethods-and-Future-Opportunities/. [Last accessed on 04 Nov 2019]

20. Rao RNG, Hadi MA, Panchal HA. A novel approach to sustained montelukast sodium release: differentially coated mini-tablets in HPMC capsules. Int J Pharm Biol Sci 2011;2:90-7.

21. Mounika A, Sirisha B, Rao V, Maheshwar U. Pharmaceutical mini tablets, its advantages and different enteric coating processes. World J Pharm Pharm Sci 2015;4:523-41

22. De Souza DF, Goebel K, Andreazza IF. Development of enteric coated sustained release minitablets containing mesalamine. Brazilian J Pharm Sci 2013;49:529-36.

23. https://ima.it/pharma/directly-compressed-mini-tabletscoated-in-a-solid-wall-pan-for-the-sustained-release-of-drugs/. [Last accessed on 16 Dec 2019]

24. https://www.researchgate.net/publication/266330162_Sustai ned_release_drug_delivery_system_A_review. [Last accessed on 10 Jul 2020]

25. Solanki B, Patel R, Barot B, Parejiya P, Shelat P. Multiple unit dosage forms: a review. Pharmtechmedica 2012;1:11-21.

26. Leon L. The theory and practice of industrial pharmacy. $2^{\text {nd }}$ ed. Varghesed publishing house: Bombay; 1991.

27. Gupta A, Bilandi A, Kataria MK, Khatri N. Tablet coating techniques: concept and recent trends. Int Res J Pharm 2012;3:50-8.

28. Paul NAC, Ernest AAA, Jim WA. Design and evaluation of chronotherapeutic pulsatile drug delivery system of cilnidipine. Universal J Pharm Res 2017;2:18-22.

29. M Tomuta I, Leucuta S. Identification of critical formulation variables for obtaining metoprolol tartrate mini-tablets. Farmacia 2010;58:719-27.

30. T N, Rao V, Hadi MA. Design and characterization of twice daily minitablets formulation of pregabalin. Int J Pharm Pharm Sci 2013;5:1484-91. 
31. Sirisha MB, Rao V, Maheshwar U. Formulation and evaluation of fenofibric acid delayed release mini tablets in capsule. Int Innovative Pharm Sci Res 2015;3:1290-304.
32. Honmane SM, Dange YD, Osmani RAM, Jadge DR. General consideration of design and development of dosage forms: preformulation review. Asian J Pharm 2017;11:479-85. 